\title{
CONTROLADOR REPETITIVO PARA INVERSORES PWM COM REFERÊNCIA DE FREQÜÊNCIA VARIÁVEL
}

\author{
Leandro Michels* \\ michels@ieee.org
}

\author{
Márcio Stefanello ${ }^{\dagger}$ \\ marciosteieee.org
}

\author{
Hilton A. Gründling ${ }^{\dagger}$ \\ ghiltonectlab.ufsm.br \\ *Universidade do Estado de Santa Catarina \\ Centro de Ciências Tecnológicas \\ Núcleo de Processamento de Energia Elétrica \\ Campus Universitário Prof. Avelino Marcante s/n, Bom Retiro, CEP 89223-100 \\ Joinville - SC - Brasil \\ ${ }^{\dagger}$ Universidade Federal de Santa Maria \\ Grupo de Eletrônica de Potência e Controle \\ Av. Roraima s/n, Camobi, CEP 97105-900 \\ Santa Maria - RS - Brasil
}

\section{ABSTRACT}

Repetitive controllers have demonstrated efficacy in the rejection of cyclic disturbances in pulse-width modulated voltage-source inverters. However, the performance of these controllers is compromised if their period are not synchronized with the period of the reference signal. This drawback restrict their use in variable frequency applications. In order to mitigate this problem, it is proposed a modification in these controllers to make them suitable for sinusoidal references with variable frequency. The proposed algorithm is simple given that is only performed a chance in the length of circular buffers and in the pointer used to address them. Experimental results, obtained with two different prototypes, are presented to validate the proposed technique.

KEYWORDS: digital control, repetitive control, PWM inverters.

\footnotetext{
Artigo submetido em 04/02/2009 (Id.: 00944)

Revisado em 19/03/2009

Aceito sob recomendação do Editor Associado Prof. Darizon Alves de Andrade
}

\section{RESUMO}

Controladores repetitivos têm se mostrado eficazes na rejeição de distúrbios cíclicos em inversores de tensão modulados por largura de pulso. Contudo, observa-se que estes controladores têm seu desempenho comprometido caso seu período não seja sincronizado com o período do sinal de referência, o que restringe o seu uso em aplicações de frequiência variável. Para mitigar este problema, é proposta uma modificação nestes controladores para adequá-los à aplicações com referência senoidal de freqüência variável. $\mathrm{O}$ algoritmo proposto é simples, sendo apenas ajustados o comprimento das memórias circulares e os ponteiros empregados para endereçá-las nos controladores repetitivos convencionais. Resultados experimentais, obtidos em dois protótipos com especifiações distintas, são apresentados para validar a técnica proposta.

PALAVRAS-CHAVE: controle digital, controle repetitivo, inversores PWM. 


\section{INTRODUÇÃO}

Os inversores de tensão modulados por largura de pulso (PWM - pulse-width modulation) são amplamente empregados em diversas aplicações, tal como em Fontes Ininterruptas de Energia (UPS - Uninterruptible Power System) e Fontes CA de Potência (AC Power Sources) (ANSI/IEEE Std. 5191981, 1981). Nestas aplicações, as especificações de projeto geralmente exigem uma alta capacidade de rejeição de distúrbios, o que demanda um estágio de saída com baixa impedância.

Devido aos requisitos de desempenho exigidos pela aplicação (IEC 62040-3, 1999), estes inversores usualmente operam com uma malha de controle de elevado desempenho. Algumas técnicas de controle discreto baseadas em realimentação instantânea têm sido utilizadas em inversores, tais como deadbeat (Mattavelli, 2005), retroação de estados (Ryan et al., 1997), entre outras. Mesmo empregando estes controladores, há casos onde não é possível se reduzir suficientemente a impedância de saída do inversor, o que resulta em valores inaceitáveis de distorção harmônica total (THD - total harmonic distortion) na tensão de saída para alguns tipos de cargas não-lineares.

A utilização de uma malha de ação instantânea em conjunto com uma lei de controle repetitiva permite uma redução substancial na impedância de saída de inversores de tensão (Rech et al., 2003). Neste esquema de controle com duas malhas, a realimentação instantânea compensa os transitórios de carga e o controlador repetitivo (RP) compensa os erros de regime permanente devido às cargas não-lineares cíclicas (Michels and Gründling, 2005).

O controle repetitivo (Hara et al., 1988; Tomizuka et al., 1989) tem como base o princípio do modelo interno (Francis and Wonham, 1975). Diferentes estruturas de controladores repetitivos (RP) foram desenvolvidas para as mais variadas aplicações (Kempf et al., 1993). Nas aplicações em inversores PWM (Haneyoshi et al., 1988), o algoritmo mais utilizado é o filtro Q, devido a baixa complexidade do código necessária para sua implementação, sua baixa demanda computacional e sua rápida taxa de convergência. Sua principal restrição é exigência de sincronismo perfeito do período da referência e dos distúrbios com um número inteiro de períodos de amostragem da lei de controle (Tomizuka et al., 1989).

Nas aplicações de inversores PWM em que a referência é uma senóide de freqüência fixa, os distúrbios provocados pelas cargas não-lineares são cíclicos e sincronizados com a referência (Michels and Gründling, 2005). Portanto, a hipótese anteriormente apresentada pode ser facilmente garantida através do projeto adequado do controlador repetitivo (Haneyoshi et al., 1988; Rech et al., 2003; Zhang et al., 2003; Marafão et al., 2004; Michels and Gründling,

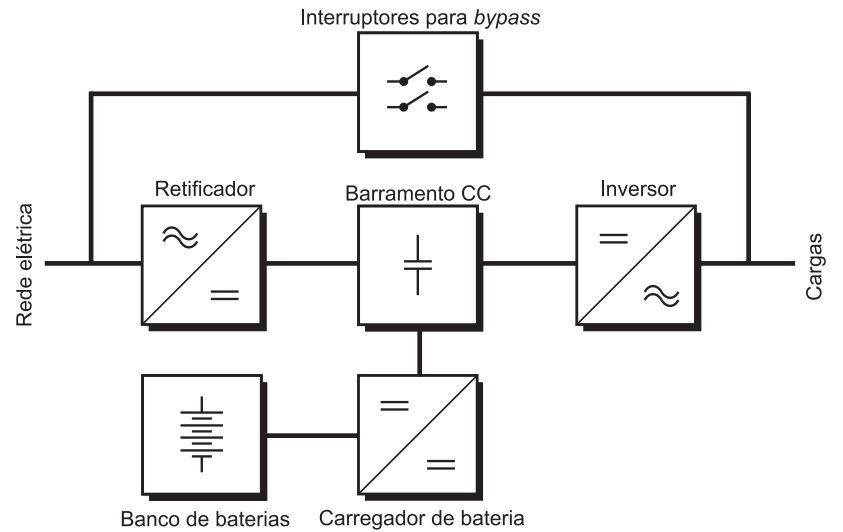

Fig. 1: UPS de dupla conversão com chave de transferência estática.

2005; Botterón and Pinheiro, 2006; Escobar et al., 2007; Ye et al., 2006; Zhou et al., 2006).

Contudo, as aplicações de inversores em que a referência é uma senóide de freqüência variável são também bastante usuais. Por exemplo, em ensaios de equipamentos eletrônicos, emprega-se fontes CA de potência para avaliar o impacto de variações da amplitude e da frequência da tensão de alimentação no funcionamento dos mesmos. Em UPS de dupla-conversão (ou UPS online), cujo diagrama em blocos é mostrado na Fig 1, se opera em dois modos: i) modo normal, onde a tensão é fornecida pelo inversor; ii) e o modo bypass, onde a energia é fornecida diretamente pela rede elétrica (IEC 62040-3, 1999). Para que a tensão de saída comute de forma suave e com tempo nulo de transferência entre os modos, é preciso garantir-se o sincronismo entre a tensão gerada pelo inversor e a componente harmônica fundamental da rede CA. Isto somente é possível através do ajuste da frequiência da referência de tensão do inversor. Para estas duas aplicações, os controladores repetitivos convencionais não são adequados porque não há sincronismo entre a referência/distúrbios e a ação de controle repetitiva.

Os controladores RP para referências de freqüência variável são discutidos em vários artigos de teoria de controle (Steinbuch, 2002; Gupta and Lee, 2006) e mecatrônica (Manayathara et al., 1996; Cao and Ledwich, 2002; Chang et al., 2006). Contudo, apesar de existir um vasto conjunto de aplicações em inversores PWM com esta característica, há poucos estudos na literatura que abordam o problema (Mattavelli and Marafão, 2004; Tzou et al., 1997; Jung et al., 1998; García-Cerrada et al., 2007; Wang et al., 2007). Muitos destes artigos apenas comentam o problema, mas não apresentam uma análise detalhada. Em Mattavelli and Marafão (2004) é sugerida a utilização de um identificador de período para ajustar a taxa de amostragem do sistema e assim se garantir o sincronismo. Em Tzou et al. (1997) é ajustado o 
comprimento das memórias (buffers) circulares para obter-se o sincronismo, sendo este realizado a partir do arredondamento do número de amostras do período anterior. Recentemente foi proposto o ajuste da frequiência em coordenadasdq para garantir o número inteiro de períodos de amostragem (García-Cerrada et al., 2007) e um controlador RP de atraso fracionário (Wang et al., 2007).

Este artigo propõem uma modificação no controle repetitivo convencional, cujo foco são as aplicações em UPS de dupla alimentação com bypass e fontes CA de potência para emulação de redes de energia. Nestas aplicações, assume-se que o sinal de referência é senoidal com uma freqüência base fixa, podendo assumir pequenas e lentas variações em torno do seu valor base. $\mathrm{O}$ algoritmo proposto ajusta o comprimento dos buffers circulares empregando uma estratégia similar a descrita em Tzou et al. (1997). Contudo, ao invés de realizar uma interpolação para o cálculo da lei de controle, o algoritmo proposto efetua apenas o ajuste dos ponteiros dos buffers circulares. Como resultado, obtém-se um algoritmo de simples implementação e baixo esforço computacional.

O artigo é organizado como segue. A Seção II descreve o sistema, incluindo o modelo dinâmico da planta e a estrutura do controlador repetitivo convencional. Na Seção III é analisado o problema abordado, enquanto na Seção IV é apresentada a modificação proposta para o controlador repetitivo convencional. Resultados experimentais são apresentados na Seção V para validar o controlador proposto.

\section{DESCRIÇÃO DO SISTEMA}

O inversor PWM monofásico alimentado em tensão é mostrado na Fig. 2, onde o inversor em ponte completa, o filtro LC e a carga são considerados a planta a ser controlada (Michels et al., 2005). As cargas conectadas aos inversores PWM são usualmente equipamentos eletrônicos, tal como computadores e processadores de dados, sistemas de comunicação, sistemas de instrumentação e controle de processos e equipamentos biomédicos. Estes equipamentos normalmente possuem, na entrada de sua fonte de alimentação, um retificador não-controlado com filtro capacitivo ou um conversor com correção de fator de potência. Por este motivo, o desempenho de inversores PWM é avaliado, neste trabalho, empregando-se um circuito que emule tais cargas, como propõe a norma IEC 62040-3 (IEC 62040-3, 1999).

A seguir, é apresentado o modelo dinâmico do inversor e a estrutura de controle considerada no presente trabalho.

\subsection{Modelo Dinâmico da Planta}

O modelo dinâmico do inversor, mostrado na Fig. 2, é nãolinear devido a carga considerada. Para linearizar este mo-

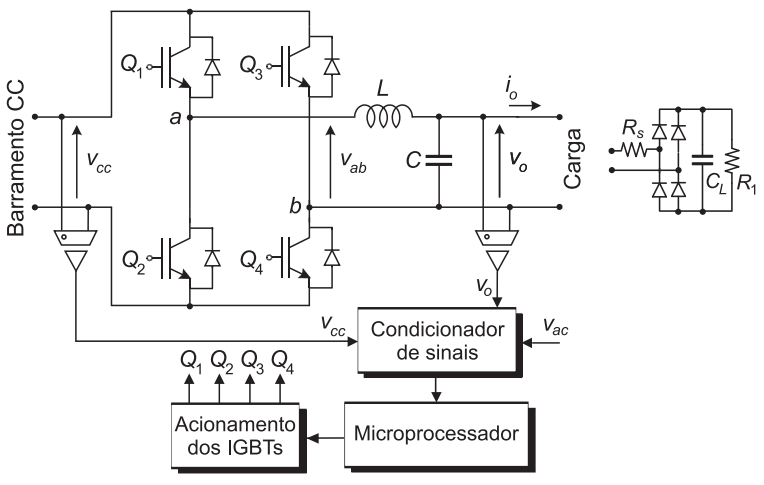

Fig. 2: Inversor PWM monofásico com controle digital.

delo, considera-se que a corrente de carga $i_{o}$ é um distúrbio exógeno (Haneyoshi et al., 1988; Rech et al., 2003; Zhang et al., 2003; Michels and Gründling, 2005; Escobar et al., 2007; Zhou et al., 2006). São assumidas as seguintes hipóteses: i) os interruptores são acionados e bloqueados apenas uma vez durante cada período de amostragem $T_{s}$, tal que neste intervalo $v_{a b}(t)$ seja um pulso de tensão de amplitude $V_{c c}$ ou $-V_{c c}$ e largura $\Delta T$; ii) a freqüência de comutação é muito maior que a máxima freqüência do sinal de entrada $u$; iii) os harmônicos na tensão de saída $v_{o}$, associados à modulação, são significativamente atenuados. Como $v_{a b}(t) \simeq u(t)$, o sistema pode ser representado pelo seguinte modelo linear e invariante no tempo:

$$
\begin{gathered}
V_{o}(s)=G_{p}(s) V_{a b}(s)+G_{d}(s) I_{o}(s), \\
G_{p}(s)=\frac{V_{o}(s)}{V_{a b}(s)}=\frac{s C r_{C}+1}{s^{2} L C+s C\left(r_{L}+r_{C}\right)+1}, \\
G_{d}(s)=\frac{V_{o}(s)}{I_{o}(s)}=\frac{s\left(C r_{C}^{2}+L\right)+r_{L}-r_{C}}{s^{2} L C+s C\left(r_{L}+r_{C}\right)+1},
\end{gathered}
$$

onde $G_{p}(s)$ é a função de transferência da planta, $G_{d}(s)$ é a função de transferência da impedância de saída e $r_{C}$ e $r_{L}$ são, respectivamente, a resistência série equivalente do capacitor $C$ e do indutor $L$ mostrados na Fig. 2.

Para obtenção das funções de transferência discretas de (2) e (3), são assumidas as seguinte hipóteses: i) a ação de controle $v_{a b}(t)$ é gerada por meio de um modulador por largura de pulso implementado digitalmente, cujo comportamento é similar ao de um retentor de ordem zero ( $\mathrm{ZOH})$; ii) o distúrbio de entrada $i_{o}(t)$ pode ser aproximado por sinal amostrado por um ZOH; iii) $v_{a b}(t), i_{o}(t)$ e $v_{o}(t)$ são amostrados simultaneamente com a frequiência $f_{s}$ constante. Como resultado, a função de transferência discreta em no domínio- $z$ de (2) e (3) são dadas, respectivamente, por:

$$
G_{p}(z)=\frac{b_{1} z+b_{0}}{z^{2}+a_{1} z+a_{0}},
$$




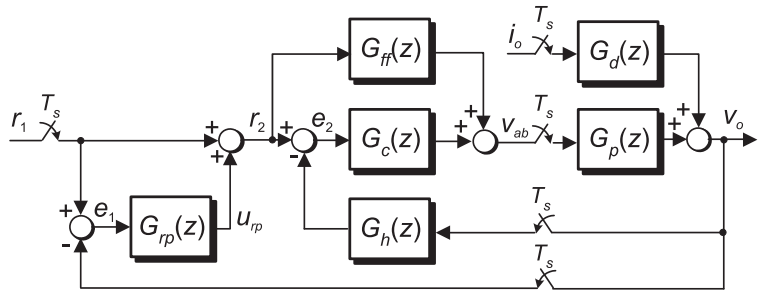

Fig. 3: Estrutura do controlador em tempo discreto.

$$
G_{d}(z)=\frac{d_{1} z+d_{0}}{z^{2}+a_{1} z+a_{0}}
$$

\subsection{Estrutura do Controlador}

A Fig. 3 mostra a estrutura do controlador do inversor monofásico. O controlador possui uma estrutura de duas camadas, sendo constituída por uma malha de realimentação instantânea e outra para compensação em regime permanente de cargas não-lineares cíclicas. Esta estrutura foi escolhida por permitir a análise de diferentes tipos de controladores, tais como o PID (proporcional-integral-derivativo), o deadbeat, por modelo de referência, por alocação de pólos, entre outros, com ou sem ação de alimentação à frente (feedforward).

O sinal de referência $r_{1}$ para o sistema de controle do inversor é senoidal com freqüência $f_{1}$. Em fontes ca de potência, este sinal é gerado internamente pelo software empregado para a realização dos ensaios. Já em UPS de dupla convesão, este sinal é gerado por um algoritmo de sincronismo a partir da tensão ca medida (Marafão et al., 2005; Cardoso et al., 2006).

A função de transferência em malha fechada entre a referência $r_{2}$ e a saída $v_{o}$ é dada por:

$$
G_{m}(z)=\frac{V_{o}(z)}{R_{2}(z)}=\frac{G_{p}(z)\left[G_{f f}(z)+G_{c}(z)\right]}{1+G_{p}(z) G_{c}(z) G_{h}(z)} .
$$

Neste artigo, foi utilizado um controlador PD com ação feedforward na malha de realimentação instantânea. Este controlador emprega a estrutura $G_{f f}(z)=G_{h}(z)=1 \mathrm{e}$ $G_{c}(z)=k_{1} z^{-1}+k_{2} z^{-2}$. Esta lei de controle é implementada pela seguinte equação de diferenças discretas:

$$
v_{a b}{ }^{*}(k+1)=k_{1} e_{2}(k)+k_{2} e_{2}(k-1)+r_{2}(k+1),
$$

onde $e_{2}(k)=r_{2}(k)-v_{o}(k)$.

A função de transferência da ação RP com $n=f_{s} / f_{1}(n \in$ $\mathbb{N}^{+}$) amostras por período do sinal de referência é dada por:

$$
G_{r p}(z)=\frac{U_{r p}(z)}{E_{1}(z)}=\frac{1}{z^{n}-Q(z)} C(z),
$$

onde $Q(z)$ e $C(z)$ são filtros utilizados para aumentar a margem de estabilidade e compensar o atraso de fase de $G_{m}(z)$, respectivamente (Hara et al., 1988). Várias estruturas podem ser usadas para $Q(z)$ e $C(z)$ (Michels and Gründling, 2005). Para simplificar a análise, neste trabalho são utilizados os filtros $Q(z)=q_{r}$ e $C(z)=c_{r} z^{d}$, onde $q_{r} \leq 1, c_{r}$ é o ganho da ação repetitiva e $z^{d}$ é o filtro usado para compensar o atraso de fase de $G_{m}(z)$. Este controlador RP é implementado por meio da seguinte equação diferenças discretas:

$$
u_{r p}(k+1)=q_{r} u_{r p}(k-n+1)+c_{r} e_{1}(k-n+d+1),
$$

onde $e_{1}(k)=r_{1}(k)-v_{o}(k)$. Assim, a referência para a malha de realimentação instantânea é dada por:

$$
r_{2}(k+1)=r_{1}(k+1)+u_{r p}(k+1) .
$$

A implementação prática da ação repetitiva utiliza memórias do tipo primeiro a entrar, primeiro a sair (FIFO - first in, first $o u t)$. Estas memórias são implementadas nos microprocessadores empregando-se memórias circulares (ou buffers circulares). Assim, o número de posições de memórias empregados para armazenar $u_{r p}$ e $e_{1}$ têm tamanho finito $n$. Contudo, (11) deve assumir a seguinte forma:

$$
u_{r p}=q_{r} u_{r p}(p)+c_{r} e_{1}(q),
$$

onde $p$ e $q$ são os ponteiros usados para o endereçamento das posições, nas memórias circulares, correspondentes aos índices $k-n+1$ e $k-n+d+1$ em (10), respectivamente. Os ponteiros são incrementados a cada período de amostragem, sendo seus valores reinicializados para 1 quando o seu valor ultrapassar $n$. No início da execução do programa, o valores destes ponteiros devem ser $p=1$ e $q=d+1$.

\section{IMPACTO DA VARIAÇÃO DA FREQÜÊN- CIA EM CONTROLADORES REPETITI- VOS}

O controlador RP convencional apresentado na Seção 2.2 não é adequado para os casos onde os distúrbios e/ou referência possuem período variável. Entretanto, nas aplicações de inversores PWM consideradas neste trabalho, o período do sinal da referência depende do comportamento da rede elétrica onde a UPS está conectada ou da frequiência gerada pela fonte CA de potência.

Por este motivo, considerou-se neste trabalho as especificações estabelecidas na norma IEC 62040-3 (1999) para UPS de dupla conversão com chave de transferência estática. Neste caso, assume-se que a referência: i) não apresenta desvios súbitos de fase; ii) possui variação de frequiência máxima de $\pm 2 \%$ com relação ao valor base (condição nominal); e iii) que a taxa máxima de variação é de $1 \mathrm{~Hz} / \mathrm{s}$.

As hipóteses assumidas por esta norma são condições extremas verificadas em redes CA. Um exemplo real mostrando 


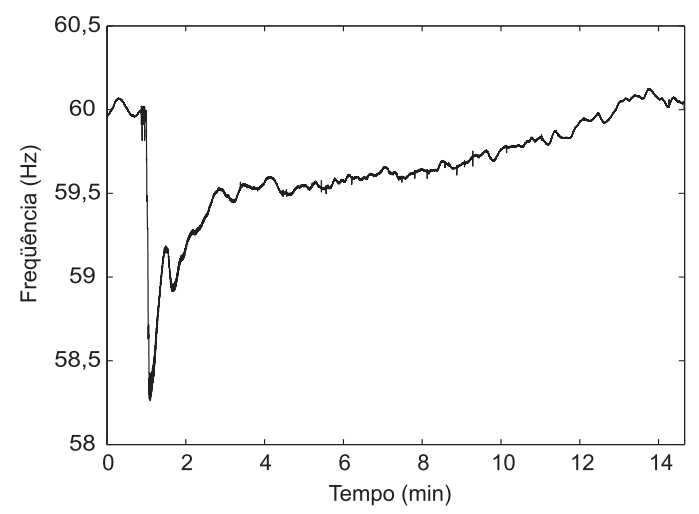

Fig. 4: Freqüência do sistema interligado nacional em 16/08/2005 entre 8h37min e 8h55min.

uma cenário em que uma grande variação pode ocorrer é apresentado em Decker et al. (2006). Neste estudo, analisouse o comportamento da frequiência do sistema elétrico brasileiro por um longo período de tempo. Durante as condições de operação normal, os resultados mostram que a freqüência do sistema oscila em torno de $0,02 \mathrm{~Hz}$ em todos os períodos de carga. Por outro lado, a Fig. 4 ilustra um exemplo onde a frequiência sofre grandes variações. Esta figura mostra o intervalo em que houve a interrupção na linha de transmissão de $765 \mathrm{kV}$ entre Itaipú e São Paulo. Esta falta, ocorrida em 16 de outubro de 2005 às 20:38 horas, resultou em uma variação na freqüência do sistema de $60,12 \mathrm{~Hz}$ para $58,32 \mathrm{~Hz}$ em $3 \mathrm{~s}$.

O impacto da variação da frequiência do sinal de referência no desempenho de controladores repetitivos é analisado a partir de resultados de simulação. Considerou-se o inversor representado na Fig. 2, cujos parâmetros são dados na Tabela 1 (Sistema A). A estrutura de controle é similar a mostrada na Seção 2.2, onde é empregado um controlador de ação instantânea do tipo PD-feedforward em conjunto com um controlador repetitivo. Os parâmetros de controle são representados na mesma Tabela.

A avaliação do desempenho foi realizada para um sinal de referência com frequiência $f_{1}$ de valor base $60 \mathrm{~Hz}$, onde uma taxa de variação de $0,2 \mathrm{~Hz} / \mathrm{s}$ foi assumida. Este valor é muito menor que o máximo definido na norma IEC 62040-3 (1999). A Fig. 5(a) mostra o erro entre a referência $r_{1}$ e a tensão de saída $v_{o}$ para $f_{1}$ variando de $60 \mathrm{~Hz}$ para $59,9 \mathrm{~Hz}$. Um pequeno erro em regime permanente é obtido para $f_{1}=60 \mathrm{~Hz}$ (THD=1,3\%), como pode-se observar na Fig. 5(b). Por outro lado, o erro aumenta significativamente quando $f_{1}$ é modificado. Para ilustrar a degradação da tensão de saída, é mostrado na Fig. 5(b) a forma de onda em regime permanente para $f_{1}=59,9 \mathrm{~Hz}$ e $f_{1}=60,1 \mathrm{~Hz}$, respectivamente. Nestes casos, obtém-se valores de THD iguais a $11,1 \%$ e $11,9 \%$, respectivamente, que são muito maiores que o valor obtido para
Tabela 1: Parâmetros do Inversor PWM

\begin{tabular}{lccc}
\hline \hline & Parâmetro & Sist. A & Sist. B \\
\hline \hline \multirow{4}{*}{ Inversor } & Tensão no barramento CC $\left(v_{c c}\right)$ & $200 \mathrm{~V}$ & $200 \mathrm{~V}$ \\
PWM & Potência nominal de saída $\left(P_{o}\right)$ & $1 \mathrm{kVA}$ & $1 \mathrm{kVA}$ \\
& Freqüência de amostragem $\left(f_{s}\right)$ & $6 \mathrm{kHz}$ & $18 \mathrm{kHz}$ \\
& Freqüência de comutação $\left(f_{s w}\right)$ & $6 \mathrm{kHz}$ & $18 \mathrm{kHz}$ \\
\hline \multirow{2}{*}{ Referência } & Amplitude eficaz $\left(v_{r e f}\right)$ & $110 \mathrm{~V}$ & $110 \mathrm{~V}$ \\
& Freqüência base $\left(f_{1}^{*}\right)$ & $60 \mathrm{~Hz}$ & $60 \mathrm{~Hz}$ \\
\hline \multirow{4}{*}{ Filtro } & Indutor do filtro $(L)$ & $1 \mathrm{mH}$ & $0,4 \mathrm{mH}$ \\
$L C$ & Resistência do indutor $\left(r_{L}\right)$ & $0,1 \Omega$ & $0,1 \Omega$ \\
& Capacitor do filtro $(C)$ & $35 \mu \mathrm{F}$ & $25 \mu \mathrm{F}$ \\
Controle & Resistência do capacitor $\left(r_{C}\right)$ & $0,05 \Omega$ & $0,05 \Omega$ \\
PD-ff & Ganho $k_{1}$ & $-0,168$ & 0,073 \\
\hline & Ganho $k_{2}$ & $-0,014$ & $-0,337$ \\
Controle & Filtro $q_{r}$ & 0,99 & 0,99 \\
repetitivo & Ganho $c_{r}$ & 0,10 & 0,10 \\
& Compensação de fase $(d)$ & 2 & 2 \\
\hline Carga & Número de amostras $(n)$ & 100 & 300 \\
não-linear & Resistor $R_{1}$ & $28 \Omega$ & $28 \Omega$ \\
\hline \hline Fig. 2) & Capacitor $C_{L}$ & $4700 \mu \mathrm{F}$ & $4700 \mu \mathrm{F}$ \\
\hline
\end{tabular}

$f_{1}=60 \mathrm{~Hz}$. Constata-se, assim, que os controladores RP não são adequados para aplicações em inversores PWM quando $n \neq f_{s} / f_{1}, n \in \mathbb{N}^{+}$.

A degradação do desempenho em regime permanente dos inversores PWM com controladores RP convencionais podem ser explicada pela análise da impedância de saída. A Fig. 6 apresenta a impedância de saída do Sistema A (ver Tabela 1) para um sistema sem e outro com ação RP. Como pode se observar, a ação repetitiva introduz uma redução significativa na impedância de saída em uma estreita banda de freqüências em torno das harmônicas múltiplas de $f_{s} / n$. Caso todos os harmônicos do distúrbio estejam sincronizados com o período da ação RP, obtém-se uma significativa rejeição do mesmo. Contudo, quando $f_{1}$ é modificado, também ocorre uma alteração de mesma proporção nas freqüências da corrente drenada pelas cargas não-lineares. Conseqüentemente, caso $f_{1}$ seja alterado de $60 \mathrm{~Hz}$ para $60,5 \mathrm{~Hz}$, a $3^{a}$ e a $9^{a}$ harmônicas drenadas por esta carga passam de $180 \mathrm{~Hz}$ para $181,5 \mathrm{~Hz}$ e de $540 \mathrm{~Hz}$ para $544,5 \mathrm{~Hz}$, respectivamente. Nestas frequiências, como se observa em detalhe na Fig. 6, o controlador repetitivo não reduz a impedância de saída. Por este motivo, os controladores repetitivos convencionais não são adequados para as aplicações em inversores PWM com referência de freqüência variável. 

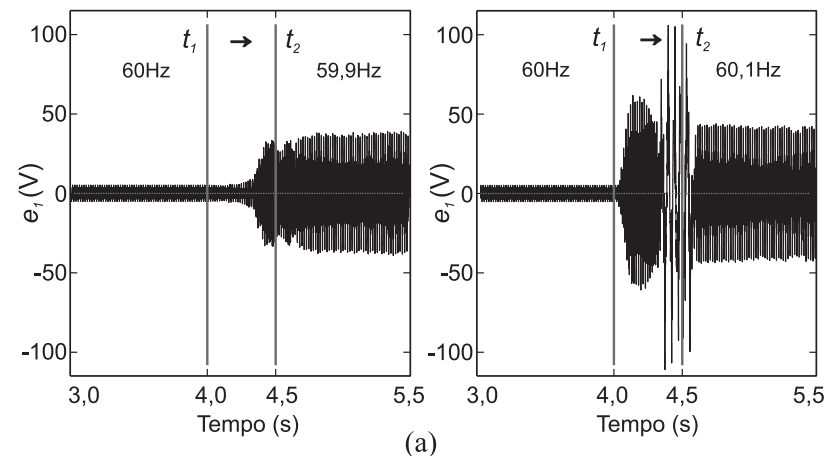

(a)
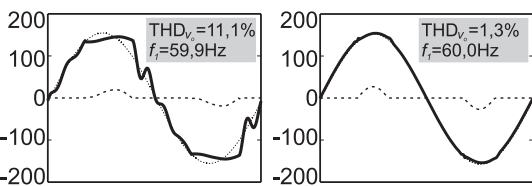

(b)

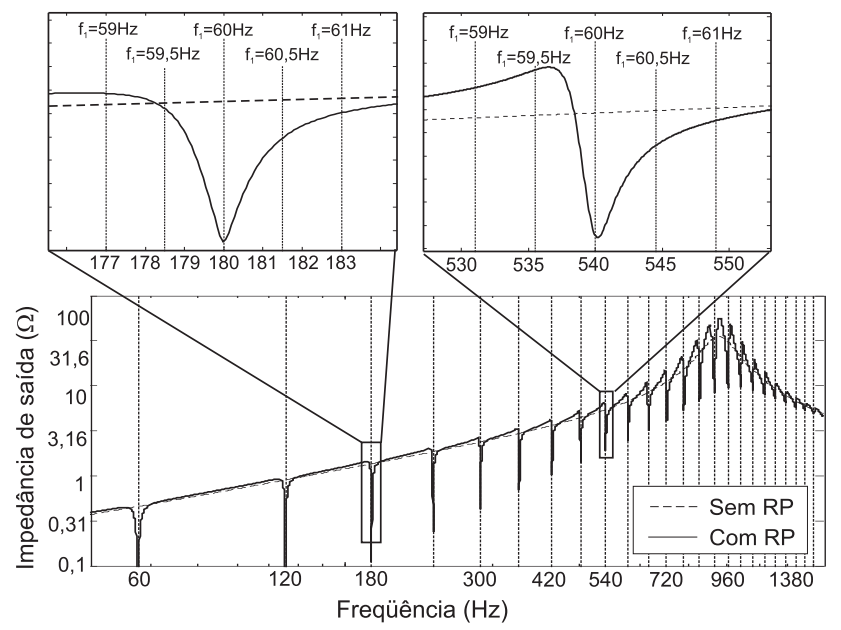

Fig. 6: Impedância de saída do Sistema A.

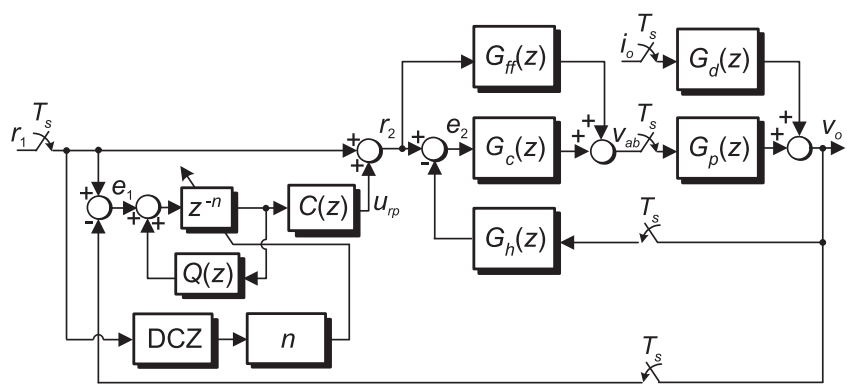

Fig. 7: Controlador RP modificado proposto.

1. Determinação da quantidade de amostras efetuadas em cada ciclo;

2. Modificação do comprimento dos buffers circulares de $u_{r p}$ e $e_{1}$ (quando for o caso);

3. Ajuste de $p$ e $q$ (quando for o caso).

O número de amostras em cada ciclo é determinado a partir da contagem do número de amostras efetuadas entre dois cruzamentos por zero, com mesma inclinação, da referência $r_{1}$. A implementação digital do detector de cruzamento por zero (DCZ) é dada por:

$$
z(k)= \begin{cases}1, & \text { se } \mathrm{r}_{1}(\mathrm{k}-1)<0 \wedge \mathrm{r}_{1}(\mathrm{k}) \geq 0 \\ 0, & \text { caso contrário }\end{cases}
$$

A condição dada em (13) é utilizada no contador do número de amostras $m$ entre dois cruzamentos por zero consecutivos:

$$
m(k)=\left\{\begin{array}{cl}
m(k-1)+1, & \text { se } \mathrm{z}(\mathrm{k})=0 \\
1, & \text { caso contrário }
\end{array} .\right.
$$
é mostrada na Fig. 7. A implementação consiste nas seguintes etapas: 


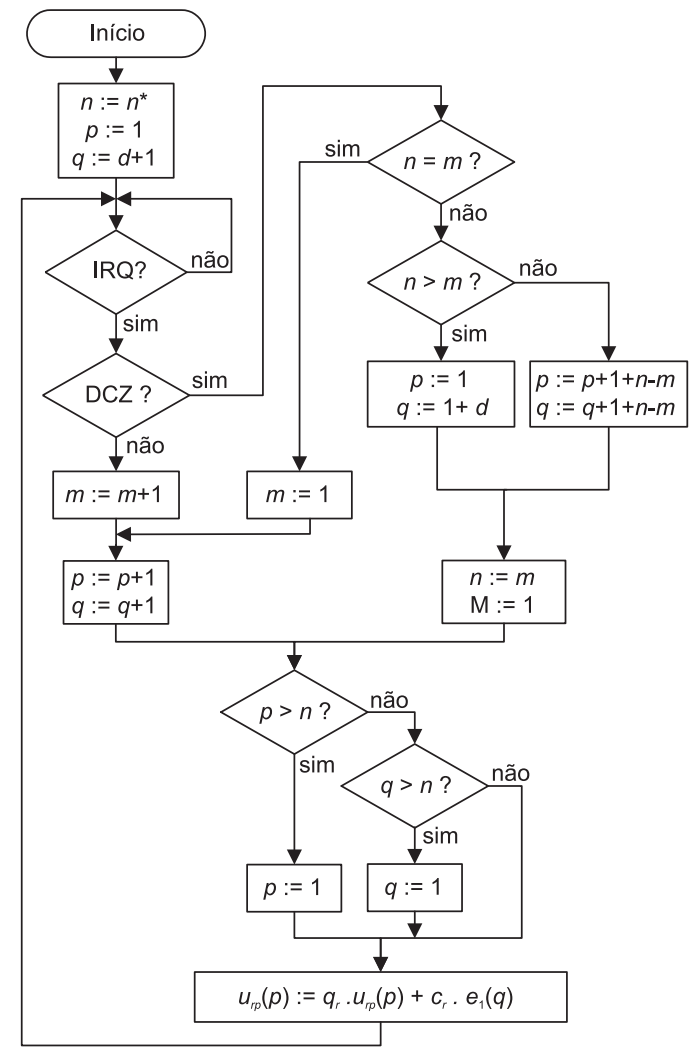

Fig. 8: Fluxograma do controlador repetitivo proposto.

Assim o número de amostras $n(k)$ é dado por:

$$
n(k)=\left\{\begin{array}{l}
m(k-1), \text { se } \mathrm{z}(\mathrm{k})=1 \\
n(k-1), \text { caso contrário }
\end{array} .\right.
$$

Observa-se que somente é possível se obter $n(k) \neq n(k-1)$ ao final de cada ciclo. Quando esta condição ocorrer, o comprimento dos buffers circulares empregados para armazenar $u_{r p}$ e $e_{1}$ são alterados de acordo com a seguinte regra:

- Se $z(k)=1$ e $n(k)<n(k-1)$, o comprimento dos buffers circulares são decrementados em $n(k-1)-n(k)$ posições, cujas posições são removidas no final do buffer. Os ponteiros são atualizados para $p=p+1 \mathrm{e}$ $q=q+1+n(k)-n(k-1) ;$

- Se $z(k)=1$ e $n(k)>n(k-1)$, o comprimento dos buffers circulares são incrementados em $n(k)-n(k-1)$ posições, incluídas no final dos buffers, cujos valores iniciais são nulos. Os ponteiros são atualizados para $p=1$ e $q=q+1+n(k)-n(k-1)$.

É importante destacar que o valor base para $n$ é dado por:

$$
n^{*}>\left\lfloor\frac{f_{s}}{f_{1}^{*}}\right\rfloor,
$$

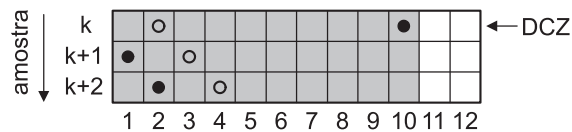

(a)

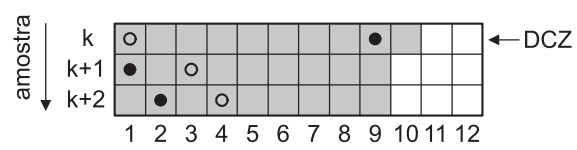

(b)

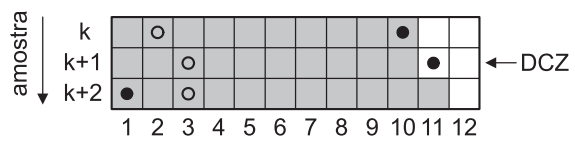

(c)

Fig. 9: Exemplo de atualização dos ponteiros dos buffers circulares. (a) $n(k)=n(k-1)$. (b) $n(k)>n(k-1)$. (c) $n(k)<n(k-1)$. Legenda: $\bullet p$, $\circ$.

e que a quantidade memória alocada para cada buffer deve ser:

$$
n_{\max }>\left\lceil\frac{f_{s}}{f_{1_{\min }}}\right\rceil .
$$

O fluxograma que descreve o algoritmo é mostrado na Fig. 8. Para ilustrar o funcionamento do mesmo, é apresentado na Fig. 9 um exemplo da evolução dos ponteiros $p$ e $q$ nos períodos posteriores à detecção de cruzamento por zero (DCZ) para $n^{*}=10, n_{\max }=12$ e $d=2$. Nesta figura, as caixas cinza e branca representam, respectivamente, as posições da memória que estão sendo usadas e não-usadas para armazenar informação de ciclos anteriores. As três condições possíveis são mostradas. Quando $n(k)=10$, como mostrado na Fig. 9(a), não há nada diferente do controlador repetitivo convencional. Entretanto, quando $n(k) \neq n(k-1)$, como mostrado na Fig. 9(b,c), os valores de $n, p$ e $q$ são alterados de acordo com a metodologia descrita nesta Seção.

\section{RESULTADOS EXPERIMENTAIS}

Duas plataformas experimentais foram desenvolvidas para uma analisar o desempenho do controlador RP proposto neste trabalho. Estas plataformas foram projetadas para emular o comportamento de inversores PWM em distintas condições de operação. A Plataforma A (Tabela 2) foi empregada para implementar o Sistema A (Tabela 1), que emula uma aplicação de inversores PWM de grande potência, cujas frequiências de comutação e amostragem são baixas. De forma similar, a Plataforma B (vide Tabela 2) foi utilizada para implementar o Sistema B (Tabela 1), que emula uma aplicação de inversores PWM de potência menor, onde as frequiências de comutação e amostragem são mais altas.

Inicialmente são apresentados os resultados obtidos em regime permanente, assumindo que a referência é senoidal com 
Tabela 2: Plataforma experimental

\begin{tabular}{lcc}
\hline \hline \multicolumn{1}{c}{ Característica } & Sistema A & Sistema B \\
\hline \hline Modulação & PWM digital & PWM digital \\
Tipo de PWM & três níveis & três níveis \\
Posição do pulso & centrado & centrado \\
\hline Chave semicondutora & IGBT & IGBT \\
Núcleo do Indutor & ferro-silício & iron powder \\
Tipo de Capacitor & polipropileno & polipropileno \\
\hline Microprocessador & PC & DSP TMS320F2812 \\
Formato dos dados & ponto flutuante & ponto fixo \\
Tamanho das variáveis & 32 bits & 32 bits \\
Conversor A/D & 12 bits & 12 bits \\
Timer do PWM & 16 bits & 16 bits \\
\hline \hline
\end{tabular}

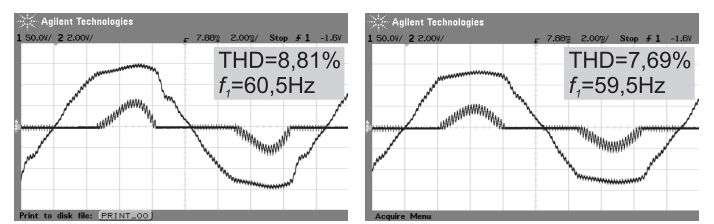

(a)

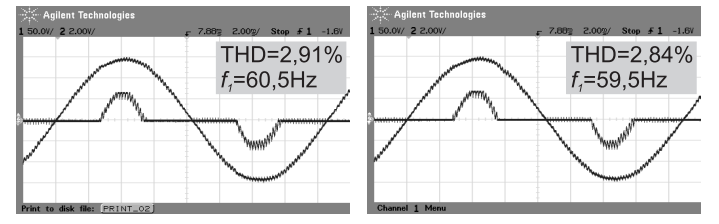

(b)

Fig. 10: Resultados experimentais obtidos em regime permanente na Plataforma A. Esquerda: $f_{1}=60,5 \mathrm{~Hz}$. Direita: $f_{1}=59,5 \mathrm{~Hz} . \quad$ (a) Algoritmo convencional. (b)Algoritmo proposto.

freqüência fixa. As Figs. 10 e 11 apresentam a forma de onda e a THD da tensão de saída. A Plataforma A foi testada para $f_{s}=6 \mathrm{kHz}$ e $f_{1}=\{60,5,59,5\} \mathrm{Hz}$, enquanto a Plataforma B para $f_{s}=18 \mathrm{kHz}$ e $f_{1}=\{58,59,60,61,62\} \mathrm{Hz}$.

As Figs. 10(a) e Fig. 11(a) mostram o comportamento em regime permanente do controlador RP convencional. O desempenho deste controlador é comprometido porque os distúrbios cíclicos não são sincronizados com o período da ação $\operatorname{RP}\left(f_{s} / f_{1} \neq n\right)$. Note que a THD é elevada quando $f_{1} \neq f_{1}^{*}$, corroborando com os resultados de simulação apresentados na Fig. 5. Por outro lado, a Fig. 10(b) e a Fig. 11(b) mostram a resposta em regime permanente do controlador RP com a modificação proposta neste artigo. Observa-se que a tensão de saída apresenta forma de onda com reduzida THD, mesmo quando $f_{1} \neq f_{1}^{*}$.
Apesar de não ter sido mostrado, verifica-se que o algoritmo proposto apresenta uma oscilação sustentada na tensão de saída. Esta oscilação é causada pelo truncamento efetuado para se ter um valor inteiro para $n$. Nos casos considerados, esta oscilação resulta numa variação cíclica da THD da ordem de $0,3 \%$, o que é pouco significativa neste caso. Contudo, tendo em vista que a amplitude desta oscilação aumenta com a diminuição de $n^{*}$, conclui-se que o algoritmo proposto pode não ser adequado em aplicações com freqüências de amostragem muito baixas.

O desempenho dos controladores repetitivos convencional e proposto, durante variações da freqüência da referência, foram analisado através do comportamento do erro entre a tensão de saída e o sinal de referência. As Plataformas A e B foram submetidas a testes onde a frequiência $f_{1}$ foi variada linearmente e sem saltos de fase a uma taxa de $1 \mathrm{~Hz} / \mathrm{s}$.

Os resultados obtidos na Plataforma A são apresentados na Fig. 12, sendo $t_{1}$ e $t_{2}$ os intervalos onde ocorreram as variações de frequiência de $59,5 \mathrm{~Hz}$ para $60 \mathrm{~Hz}$ (coluna da esquerda) e de $60,5 \mathrm{~Hz}$ para $60 \mathrm{~Hz}$ (coluna da direita). O algoritmo convencional, cujo resultado está apresentado na Fig. 12(a), apresenta erro reduzido para $f_{1}=60 \mathrm{~Hz}$. Contudo, o erro aumenta significativamente quando esta frequiência é variada, como previsto anteriormente, o que resulta em tensões de saída distorcidas como as mostradas na Fig 10(a). Por outro lado, as formas de onda do erro obtidas para o algoritmo proposto, mostradas na Fig. 12(b)), são significativamente menores que as obtidas para o algoritmo convencional. Nestes casos, o erro mantém-se praticamente inalterado, inclusive nos intervalos de tempo em que a freqüência da referência está sendo variada. Observa-se que não é possível distinguir o intervalo de tempo em que está ocorrendo a variação da frequiência, o que demonstra que a tensão de saída mantém-se pouco distorcida em qualquer situação.

A Fig. 12 apresenta o comportamento do erro obtido para a Plataforma B. Como observa-se nesta figura, a freqüência da referência foi variada entre $58 \mathrm{~Hz}$ a $62 \mathrm{~Hz}$ (coluna da esquerda) e de $62 \mathrm{~Hz}$ a $58 \mathrm{~Hz}$ (coluna da direita). Novamente, os resultados mostram uma significativa diferença entre o comportamento do erro com o controlador repetitivo convencional (Fig 12(a)) e com o controlador proposto (Fig 12(b)). Contata-se que o desempenho do algoritmo proposto não sofre uma alteração signficativa devido a variação da frequiência da referência, mesmo quando esta é alterada em $\pm 2 \mathrm{~Hz}$.

Por fim, é analisado o tempo de execução da rotina no DSP para o sistema implementado na Plataforma B. O algoritmo repetitivo convencional possui um tempo de execução de $2,61 \mu \mathrm{s}$, enquanto que proposto de $2,71 \mu \mathrm{s}$. Ou seja, o algoritmo proposto demanda apenas $4 \%$ de tempo adicional de execução, o que demonstra a sua simplicidade e eficiência. 


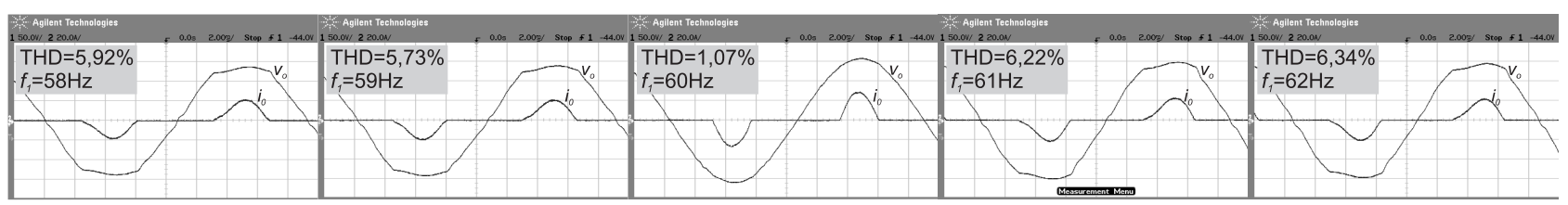

(a)

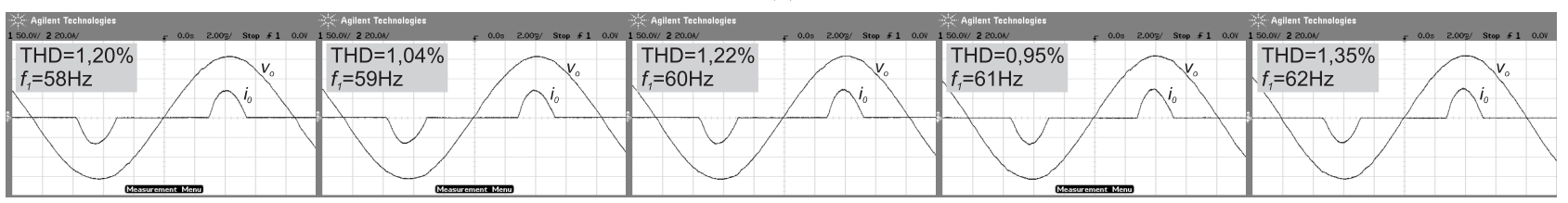

(b)

Fig. 11: Resultados experimentais obtidos em regime permanente na Plataforma B. Da esquerda para a direita: $f_{1}=58 \mathrm{~Hz}$, $f_{1}=59 \mathrm{~Hz}, f_{1}=60 \mathrm{~Hz}, f_{1}=61 \mathrm{~Hz}$ e $f_{1}=62 \mathrm{~Hz}$. (a) Algoritmo convencional. (b) Algoritmo proposto.
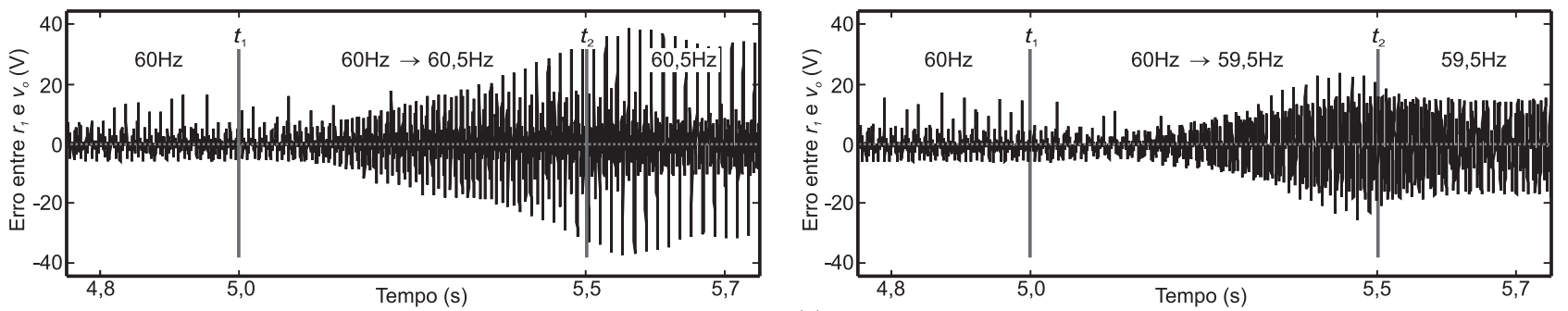

(a)
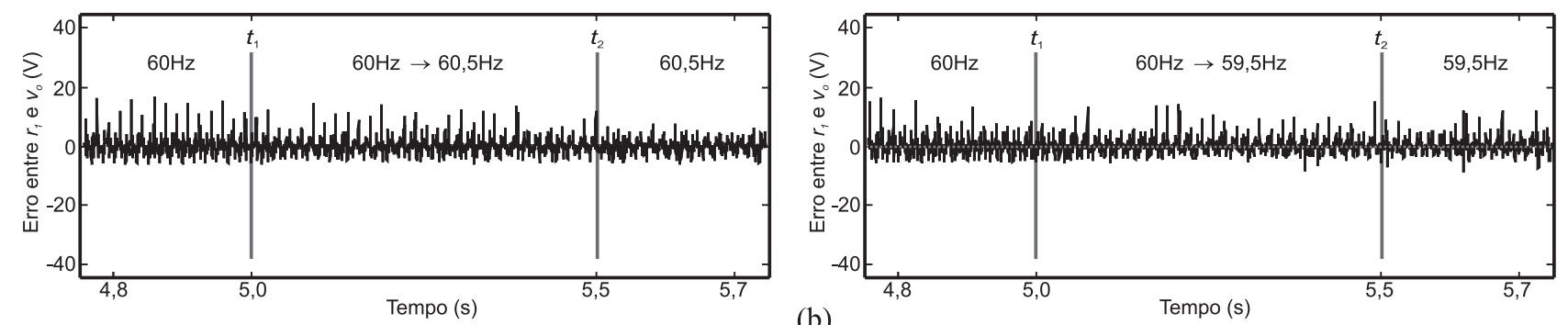

Fig. 12: Resultados experimentais para variações contínuas na freqüência da referência no Sistema A. Esquerda: $f_{1}=60 \mathrm{~Hz} \rightarrow 60,5 \mathrm{~Hz}$. Direita: $f_{1}=60 \mathrm{~Hz} \rightarrow 59,5 \mathrm{~Hz}$. (a) Algoritmo convencional. (b) Algoritmo proposto.

\section{CONCLUSÕES}

Embora os inversores PWM monofásicos com controladores repetitivos convencionais apresentem um bom desempenho em regime permanente para referências senoidais e cargas lineares e não-lineares, estes controladores possuem uma resposta insatisfatória quando o período da referência é alterado. Analisando-se o problema, mostra-se que mesmo pequenas variações neste período são suficientes para comprometer o desempenho dos controladores repetitivos. Por este motivo, este artigo propõe uma modificação nos algoritmos convencionais para torná-los adequados a rastrear referências senoidais de freqüência variável. O algoritmo proposto é simples, demanda pouco esforço computacional adicional e tem a característica de operar sem a necessidade de alterar a taxa de amostragem do sistema. Resultados experimentais, obtidos para dois sistemas com características distintas, demonstram que o controlador repetitivo proposto melhora significativamente o desempenho dos inversores PWM nestas situações.

Apesar do algoritmo de reinicialização do controlador repetitivo ter sido aqui aplicado a um inversor PWM monofásico, o mesmo pode ser usado sem restrições em sistemas trifásicos, tanto com controle em eixos estacionários quanto síncronos. Contudo, devido ao aparecimento de oscilações sustentadas indesejáveis, destaca-se que o algoritmo proposto não é adequado para sistemas onde a referência possui freqüências próximas à frequiência de amostragem. 

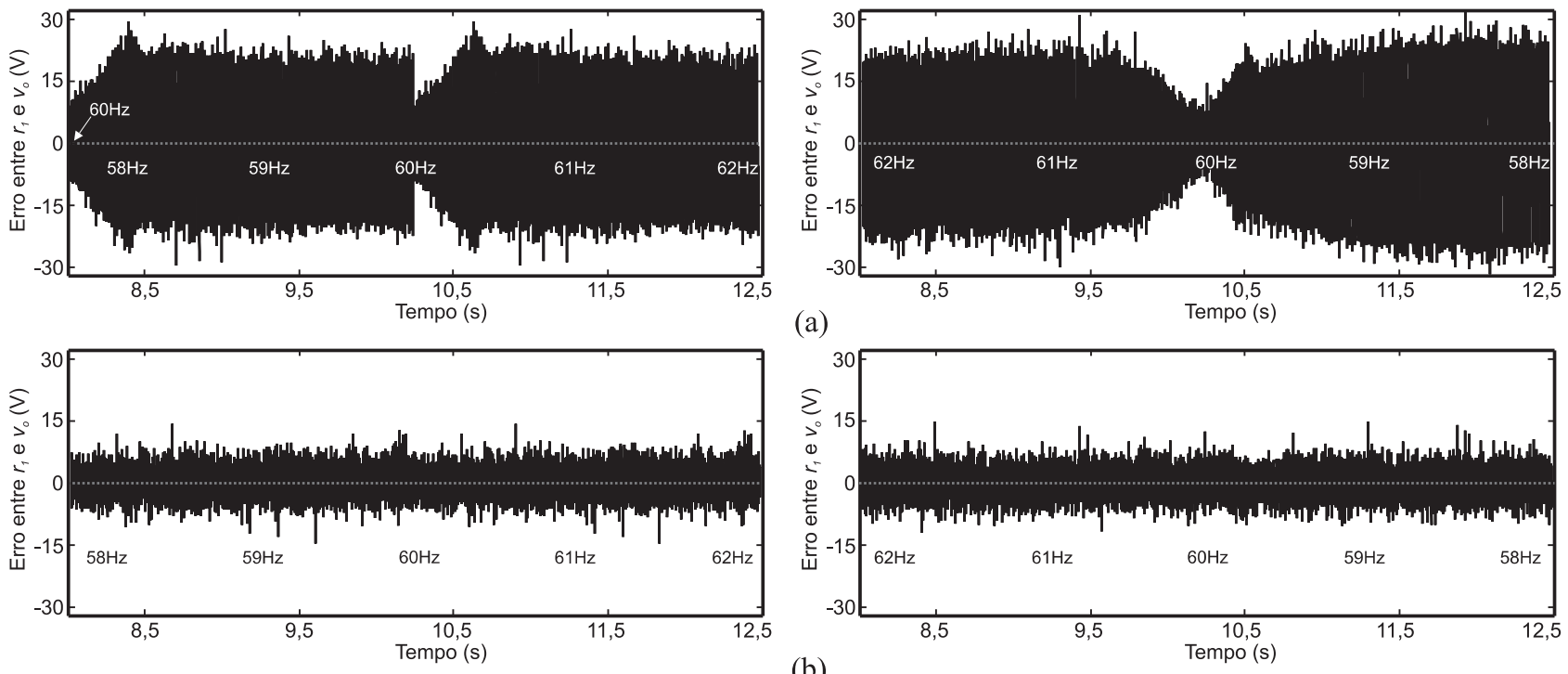

Fig. 13: Resultados experimentais para variações contínuas na freqüência da referência no Sistema B. Esquerda: $f_{1}=58 \mathrm{~Hz} \rightarrow 62 \mathrm{~Hz}$. Direita: $f_{1}=62 \mathrm{~Hz} \rightarrow 58 \mathrm{~Hz}$. (a) Algoritmo convencional . (b) Algoritmo proposto.

\section{REFERÊNCIAS}

ANSI/IEEE Std. 519-1981 (1981). IEEE Guide for Harmonic Control and Reactive Compensation of Static Power Converters, American National Standards Institute. Washington D.C., EUA.

Botterón, F. and Pinheiro, H. (2006). Controlador discreto de alto desempenho com base num modelo interno em eixos síncronos para UPS trifásica que satisfaz a norma IEC62040-3, Sobraep Eletrônica de Potência 11(2): 135-147.

Cao, Z. and Ledwich, G. F. (2002). Adaptive repetitive control to track variable periodic signals with fixed sampling rate, IEEE Trans. Contr. Syst. Technol. 7(3): 378384.

Cardoso, R., de Camargo, R. F., Pinheiro, H. and Gründling, H. A. (2006). Estruturas de sincronismo monofásica e trifásica baseadas no filtro de Kalman, SBA Controle \& Automação 17(4): 493-512.

Chang, K., Shim, I. and Park, G. (2006). Adaptive repetitive control for an eccentricity compensation of optical disk drivers, IEEE Trans. Consum. Electron. 52(2): 445450 .

Decker, I. C., Dotta, D., Agostini, M. N., Zimath, S. L. and Silva, A. S. (2006). Performance of a synchronized phasor measurements system in the Brazil power system, IEEE PES General Meet., Montreal, Canadá.
Escobar, G., Valdez, A. A., Leyva-Ramos, J. and Mattavelli, P. (2007). Repetitive-based controller for a UPS inverter to compensate unbalance and harmonic distortion, IEEE Trans. Ind. Electron. 54(1): 504-510.

Francis, B. A. and Wonham, W. M. (1975). The internal model principle for linear multivariable regulators, Appl. Math. Optimization, 2(1): 170-194.

García-Cerrada, A., Pinzón-Ardila, O., Feliu-Batlle, V., Roncero-Sánchez, P. and García-Gonzalez, P. (2007). Application of a repetitive controller for a threephase active power filter, IEEE Trans. Power Electron. 22(1): 237-246.

Gupta, M. and Lee, J. H. (2006). Period-robust repetitive model predictive control, Journal of Process Control 16(6): 545-555.

Haneyoshi, T., Kawamura, A. and Hoft, R. G. (1988). Waveform compensation of PWM inverter with cyclic fluctuating loads, IEEE Trans. Ind. Appl. 24(4): 582-589.

Hara, S., Yamamoto, Y., Omata, T. and Nakano, M. (1988). Repetitive control system: A new type servo system for periodic exogenous signals, IEEE Trans. Autom. Contr. 33(7): 659-668.

IEC 62040-3 (1999). Uninterruptible Power Systems (UPS) Part 3: Method of Specifying the Performance and Test Requirements, International Electrotechnical Commission. 
Jung, S.-L., Huang, H.-S. and Tzou, Y.-Y. (1998). A threephase PWM AC-DC converter with low swithcing frequency and high power factor using DSP-based repetitive control technique, Conference Records of 29th IEEE PESC, Fukuoka, Japan, pp. 517-523.

Kempf, C., Messner, W. and Tomizuka, M. (1993). Comparison of four discrete-time repetitive control algorithm, IEEE Contr. Syst. Mag. 13(6): 48-54.

Manayathara, T. J., Tsao, T.-C., Bentsman, J. and Ross, D. (1996). Rejection of unknown periodic load disturbances in continuous steel casting process using learning repetitive control approach, IEEE Trans. Contr. Syst. Technol. 4(3): 259-265.

Marafão, F. P., Deckmann, S. M., Pomilio, J. A. and Machado, R. Q. (2005). Metodologia de projeto e análise de algoritmos de sincronismo PLL, Sobraep Eletrônica de Potência 10(1): 7-14.

Marafão, F. P., Mattavelli, P., Buso, S. and Deckmann, S. M. (2004). Repetitive-based control for selective active filters using discrete cosine transform, Sobraep Eletrônica de Potência 9(1): 29-36.

Mattavelli, P. (2005). An improved deadbeat control for UPS using disturbance observers, IEEE Trans. Power Electron. 52(1): 206-212.

Mattavelli, P. and Marafão, F. P. (2004). Repetitive-based control for selective harmonic compensation in active power filters, IEEE Trans. Ind. Electron. 51(5): 10181024.

Michels, L., de Camargo, R. F., Botterón, F. and Pinheiro, H. (2005). Metodologia de projeto de filtros de segunda ordem para inversores de tensão com modulação PWM digital, SBA Controle \& Automação 16(2): 221-242.

Michels, L. and Gründling, H. A. (2005). Procedimento de projeto de controladores repetitivos para o estágio de saída de fontes ininterruptas de energia, Sobraep Eletrônica de Potência 10(1): 39-50.

Rech, C., Pinheiro, H., Gründling, H. A., Hey, H. L. and Pinheiro, J. R. (2003). Comparison of digital control techniques with repetitive integral action for low cost PWM inverters, IEEE Trans. Power Electron. 18(1): 401-410.

Ryan, M. J., Brumsickle, W. E. and Lorenz, R. D. (1997). Control topology options for single-phase UPS inverters, IEEE Trans. Ind. Electron. 33(2): 493-501.

Steinbuch, M. (2002). Repetitive control for systems with uncertain period-time, Automatica 38(12): 2103-2109.
Tomizuka, M., Tsao, T.-C. and Chew, K.-K. (1989). Analysis and synthesis of discrete-time repetitive controllers, Trans. ASME: J. Dyn. Syst., Meas., Contr. 111: 353358.

Tzou, Y.-Y., Ou, R.-S., Jung, S.-L. and Chang, M.-Y. (1997). High-performance programmable AC power source with low harmonic distortion using DSP-based repetitive control technique, IEEE Trans. Ind. Appl. 12(4): 715-725.

Wang, Y., Wang, D., Zhang, B. and Zhou, K. (2007). Fractional delay based repetitive control with application to PWM DC/AC converters, Conference Record of the IEEE 22nd ISIC, Singapore, pp. 928-933.

Ye, Y., Zhou, K., Zhang, B., Wang, D. and Wang, J. (2006). High-performance repetitive control of PWM DC-AC converters with real-time phase-lead FIR filter, IEEE Trans. Circ. Syst. -II:Exp. Biefs 53(8): 768-772.

Zhang, K., Kang, Y., Xiong, J. and Chen, J. (2003). Direct repetitive control of SPWM inverter for UPS purpose, IEEE Trans. Ind. Appl. 18(3): 784-792.

Zhou, K., Low, K.-S., Wang, D., Luo, F.-L., Zhang, B. and Wang, Y. (2006). Zero-phase odd-harmonic repetitive controller for a single-phase PWM inverter, IEEE Trans. Power Electron. 21(1): 193-201. 